\title{
Application of Simulated Annealing for Color Pattern Recognition to Hybrid Optoelectronic Joint Transform Correlator
}

\author{
Chulung Chen, Yunghsin Hsu, Sihliang Fu, Weichih Liao and Chengsyuan You \\ Department of Photonics Engineering, Yuan Ze University, Taiwan; \\ chulung@saturn.yzu.edu.tw
}

\begin{abstract}
For invariant pattern recognition, a method using simulated annealing algorithm is introduced. Near optimal quantized reference functions are designed to be displayed on liquid crystal spatial light modulators. Mach-Zehnder joint transform correlator is adopted as the system for recognition of color targets. From numerical results, the optoelectronic pattern recognition system with simulated annealing algorithm shows a promising capability.
\end{abstract}

Keywords: Simulated Annealing; Hybrid Optoelectronic Joint Transform Correlator; Color Pattern Recognition.

\section{Introduction}

VanderLugt correlator (VLC) [1] was proposed for comparing two signals by utilising the Fourier transforming properties of a lens. In 1966, Weaver and Goodman [2] introduced the joint transform correlator (JTC) for pattern recognition application. A few years later, LCD based joint transform correlator (JTC) [3] proposed by Yu and Lu is an attractive tool for pattern recognition. Since then, the JTC configuration has received increased attention because it can be easily implemented. However, the classical JTC suffers from strong zero order term (also called DC term) and broad correlation width. The DC term is the sum of each auto-correlation of the reference image and the target image at the output of correlation plane. The value of the DC term will influence the performance, therefore the removal of the nonzero-order term is of great importance.

Lu et al. [4] utilized phase-shifting technique to design a nonzero- order JTC (NOJTC) and Li et al. [6] used the joint transform power spectrum (JTPS) subtraction strategy to realize the NOJTC. The MachZehnder JTC (MZTC) [6] can remove the zero-order term in only one step directly without storing the Fourier spectra of both the reference and target images beforehand. Later, Chen et al. $[7,8]$ adopted constraint optimization based on Lagrangian method to yield a sharp correlation peak.

In order to apply reference function in the liquid crystal spatial light modulator, quantized version of the reference template is necessary. On the other hand, the simulated annealing (SA) method $[9,10]$ have been successfully applied to optimization problems. Annealing is a physical process of decreasing temperature slowly in order to reach the global minimum energy states. We will take advantage of this feature for color pattern recognition. 


\section{Analysis}

The MZJTC structure is shown in Figure. 1. It includes one laser, one spatial filter, one collimated lens $(\mathrm{CL})$, three beam splitters (BS), three polarizing beam splitters (PBS), three Fourier lenses (FL), three reflective liquid spatial light modulators (RLCSLM), three charge coupled device (CCD) cameras, one electronic subtractor (ES) which is used for removing the zero-order term of JPTS, and one computer for controlling the whole system. There are one half wave plate (HWP) and one quarter wave plate (QWP) in front of each RLCSLM. The MZJTC structure is based on the Mach-Zehnder interferometer technique with Stokes relationships. The difference between NOJTC and MZJTC is the MZJTC structure only needs one step to remove the zero-order term of JTPS. The processes are presented as follows.

In the begnining, 3 color component of the test color image are jointly displayed in grascale at the RLCSLM1. In a similar manner, 3 color component of the test color image are displayed in grascale at the RLCSLM2. The target on the RLCSLM1 is illuminated and Fourier optically transformed by FL1. After passing through the PBS3, the irradiation of transmitted and reflected Fourier spectrum is respectively detected by CCD1 and CCD2 in the frequency domain. Then, the difference of joint Fourier power spectrum between CCD1 and CCD2 is displayed at the RLCSLM3. Finally, CCD3 captures its Fouier transform spectrum, which contains the overlapping of each cross-correlation of the reference component and the target component. Meanwhile, the zero-order term is removed. More detailed analysis of MZJTC can be found [10-12].

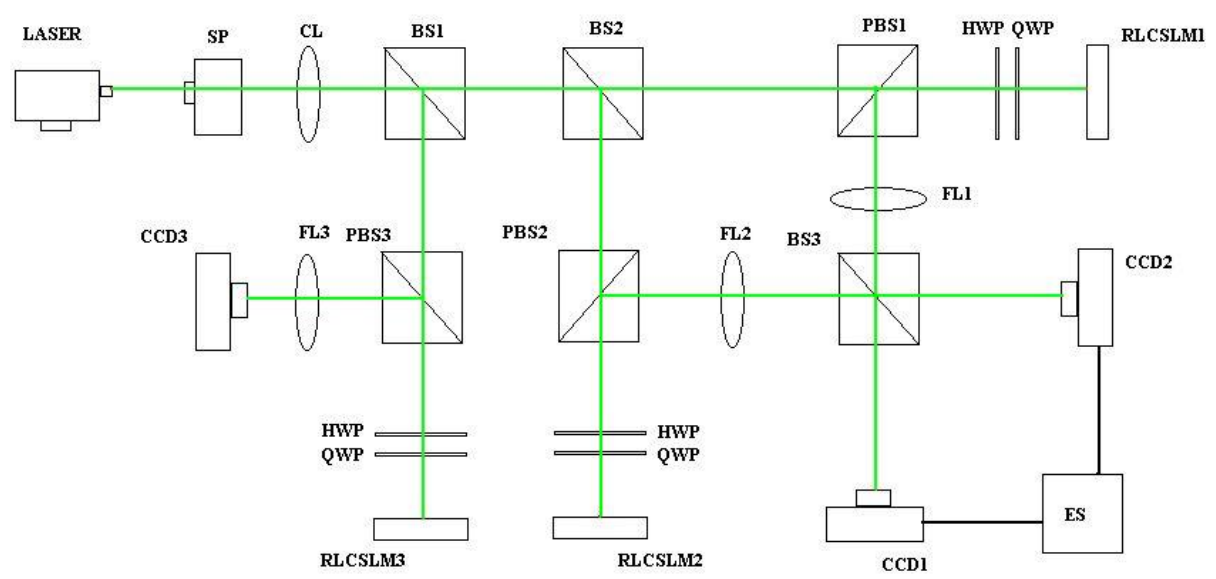

Figure 1. Mach-Zehnder joint transform correlator.

To evaluate the recognition ability, some measurement criteria [13] including correlation peak intensity (CPI) and peak to sidelobe ratio (PSR) are adopted. CPI is the cross-correlation peak intensity at the correlation output plane. PSR is the primary correlation peak energy versus secodary peak energy in the region of interest at the correlation output plane

\section{Proposed Algorithm}

One colorful butterfly is selected as the basic pattern of the target, whose size is of $64 \times 64 \times 3$ pixels. It is separated into R、G、B channels. For comparison, another butterfly is selected as the nontarget. These two images are shown in Figure 2. For simplicity, We rotate the target in plane from $-14^{\circ}$ to $14^{\circ}$, and select patterns $2^{\circ}$ apart. Totally there are 15 rotationally distorted patterns used as the training set for each color channel. Next, we utilize the training set to obtain a continuous reference function by the constraint optimization technique and then perform the quantization operation on this reference template. The purpose is to obatin a better starting solution within SA 
algorithm. In our study, the CPE (correlation plane energy) is proposed to construct the energy function.
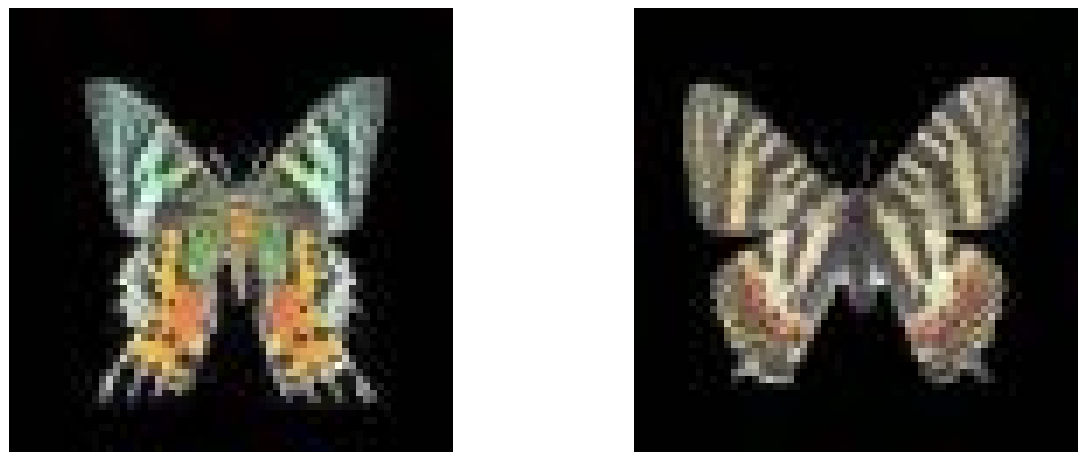

Figure 2: Target (left) and nontarget (right)

A detailed process in SA algorithm for each channel is described as follows:

o Step 1: Yield the initial reference function from constrained optimization technique.

o Step 2: Calculate CPE and CPI for each training image, compute the ratio, and add all the ratios together as the energy function $E_{\text {old. }}$. It is expressed as.

$$
E_{\text {old }}=\sum_{i=1}^{N} \frac{\mathrm{CPE}_{\mathrm{i}}}{\mathrm{CPI}_{\mathrm{i}}}
$$

Here $i$ is the index of the training image.

o Step 3: Alter the level number just for one pixel of the reference function $h(x, y)$, and then calculate the new energy function $E_{\text {new }}$.

o Step 4: If the minimum peak value of the new cross-correlation energy function for all training images is not greater than, say, 0.85 times of the minimum peak value of the old crosscorrelation energy function, the alteration of the pixel value won't be accepted and the process returns to the step 3 .

o Step 5: Calculate the difference of energy functions, which is $\Delta \mathrm{E}$ and expressed as

$$
\Delta E=E_{\text {new }}-E_{\text {old }}
$$

0 Step 6: If $\Delta E \leq 0$, accept the level number in the new reference function $\mathrm{h}(\mathrm{x}, \mathrm{y})$, set $E_{\text {new }}$ to be the next time calculated system temperature $\mathrm{T}$, which is the new starting point $E_{\text {old }}$

0 Step 7: If not, compute the probability. If it is greater than a random number in the range between 0 and 1 , and then accept the alteration of the pixel value.

o Step 8: Check whether all pixels have been operated. If they have, move to the next step. Otherwise go back to step 4.

o Step 9: Record the value of energy function in each cycle. If the normalized standard deviation of energy for the last 10 cycles is smaller than, say, 0.03 , and then terminate the computaion and exit the algorithm. Otherwise reduce system temperature by $10 \%$, and go back to step 3 . 

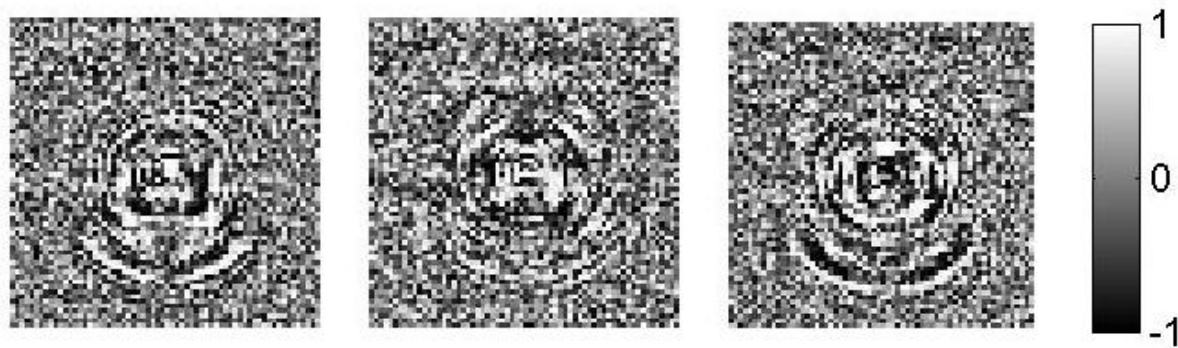

Figure 3: Three reference templates obtained by SA algorithm

\section{Result}

Figure 3 shows the 3 reference functions in grayscale using 31 levels. Each reference function corresponds to different color component. The dynamic range is also illustrated. It is worthy to notice that the CPI can not be the same for all training targets. Specifically, in our proposed technique, the minimum value of the $\mathrm{CPI}$ for these training targets is set as the threshold. Furthermore, the correlation intensity has been normalized to a range between 0 and 1 , based on the threshold. Therefore, values above the threshold CPI are set to 1 . The CPI curve versus the rotation angle for the target as well as for the nontarget are shown in Figure 4 for the sake of comparison. If the target, for examples, at $11^{\circ}$ rotation angle is not in the training set, its CPI value drops below 1 . However, the reduction is no more than $20 \%$. The target can still be detected. We can set a threshold value of correlation peak, above which the input can be treated as a target and below which it is a non-target. Therefore, performance is slightly degraded. Figure 5 indicates that the correlator yields PSR values higher than 27 for training subjects. Figure 6 shows the intensity distribution of the correlation output in the region of interest where addition of desired cross correlations of each channel occurs. Both the target and nontarget are $10^{\circ}$ rotated. As expected, high correlation peak corresponds to the correct pattern, whereas low correlation profile is observed for the nontarget. We obtain recognition of target and discrimination of nontarget.

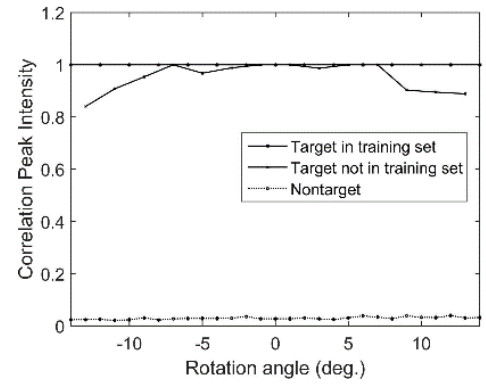

Figure 4: $\mathrm{CPI}$ versus rotation angle

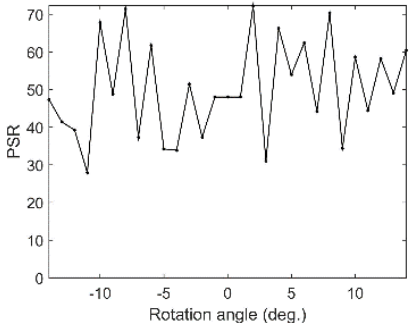

Figure 5: PSR of the target versus rotation angle
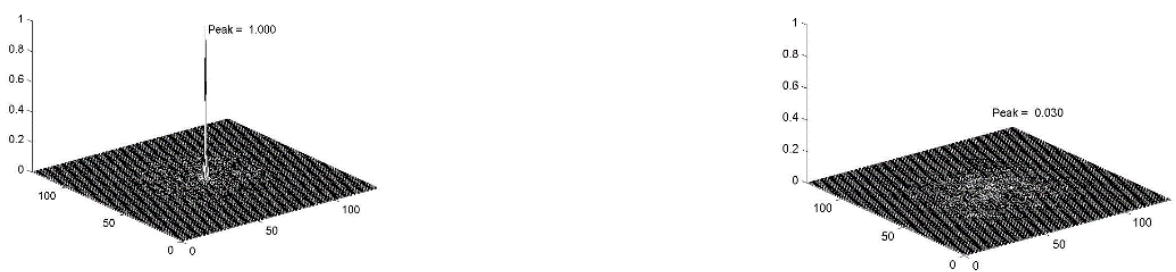

Figure 6: Sample of correlation output for target (left) and nontarget (right) rotated by $10^{\circ}$ 


\section{Conclusion}

In this paper, we have proposed a MZITC with SA for pattern recognition. The SA process will be terminated when the normalized standard deviation of energy function for the last ten iterations is smaller than some value by supposing that convergence is achieved. The performance with SA in the optoelectronic pattern recognition system is promising. The result verifies the feasibility of our proposed method.

\section{REFERENCES}

[1]. VanderLugt, A., Signal detection by complex spatial filtering. Information Theory, IEEE Transactions on, 1964. 10(2): p. 139-145.

[2]. Weaver, C. S. and J. W. Goodman, A technique for optically convolving two functions. Applied Optics, 1966. 5: p. 1248-1249.

[3]. Yu, F. T. S. and X. J. Lu, A real-time programmable joint-transform correlator. Optics Communications, 1984. 52: p. $10-16$

[4]. Lu, G., et al., Imentation of a non-zero-order joint-transform correlator by use of phase-shifting techniques. Applied Optics, 1997. 36: p. 470-483.

[5]. Li, C., S. Yin and F. T. S. Yu, Nonzero-order joint transform correlator. Optical Engineering, 1998. 37: p. 5865.

[6]. Cheng, C. and H. Tu, Implementation of a nonzero-order joint transform correlator using interferometric technique. Optical Review, 2002. 9: p. 193-196.

[7]. Wu, C., C. Chen, and J. Fang, Linearly constrained color pattern recognition with a non-zero order joint transform correlator. Optics Communications, 2002. 214: p. 65-75.

[8]. Chen C., and J. Fang, Optimal synthesis of a real-valued template for synthetic aperture radar pattern recognition. Microwave and Optical Technology Letters, 2002. 32(2): p. 91-95.

[9]. Kirkpatrick, S., et al., Optimization by simulated annealing. Science, 1983. 220: p. 671-680,

[10]. Chen, C. and C. Chen, A Mach-Zehnder joint transform correlator with the simulated annealing algorithm for pattern recognition. Optics Communications, 2011. 284: p. 3946-3953.

[11]. Fu, S., et al., Application of simulated annealing for color pattern recognition to the optoelectronic correlator with liquid crystal device. The 2012 IAENG International Conference on Imaging Engineering. p.683-688.

[12]. Liu, C., et al., Pattern recognition by Mach-Zehnder joint transform correlator with binary power spectrum," Proceedings SPIE 8559.

[13]. Kumar, B. V. K. V. and L. Hassebrook, Performance measures for correlation filters. Applied Optics, 1990. 29: p. 2997-3006. 